\title{
Experimental investigation of the failure modes of in-plane loaded inserts in CFRP sandwich panels
}

\author{
Philip Richert ${ }^{1, *}$, Athanasios Dafnis ${ }^{1}$, and Kai-Uwe Schröder ${ }^{1}$ \\ ${ }^{1}$ Institute of Structural Mechanics and Lightweight Design, RWTH Aachen University, \\ Wüllnerstraße 7, 52062 Aachen, Germany
}

\begin{abstract}
The aim of this paper is the experimental determination and investigation of the different failure modes of various insert types under inplane load in sandwich materials with CFRP face sheets and a perforated aluminum honeycomb core. In addition, also insert under an out-of-plane load are tested. In total three different kind of inserts are investigated: blind inserts, trough-the-thickness inserts and special inserts which are developed within the iBOSS research project. The inserts are tested until failure and monitored with the digital image correlation system Aramis. This test monitoring is intended to determine the first failure mode. It is shown that this works well for out-of-plane loaded insert connections and the special inserts under in-plane load. However, this does not work so well with in-plane loaded standard inserts. In further investigations, a failure of the adhesive shortly before failure of the top layers can be shown.
\end{abstract}

\section{Introduction}

Sandwich materials are often used in lightweight structures since their special geometry provides high rigidity combined with low weight. These structures generally consist of two stiff in-plane load-bearing face sheets and a light core that absorbs shear forces. Usually the sandwich structures have very good structural mechanical properties from a global perspective. But if they are loaded locally, however, only small forces are sufficient to destroy the structures. Such local loads are caused by any kind of mounting points such as screw connections. Since there is no possibility to cut a thread into the core for a screw connection, special connection points with integrated threads known as inserts have to be adhesively potted into the sandwich. The failure behavior of these insert sandwich connection is very complex.

Within the iBOSS research project, a modular satellite concept is being developed. The satellite itself consists of several components that can be coupled together. The sides of these cube-shaped building blocks consist of sandwich plates. To attach individual systems

*Corresponding author: philip.richert@sla.rwth-aachen.de 
and payloads to these surfaces, the previously mentioned inserts are required. Due to the special requirements resulting from the iBOSS concept, three different types of inserts are used. For a suitable structural design, for these inserts the failure loads and failure modes must be known.

Due to many, mostly experimental investigations, the different failure modes of these inserts under an out-of-plane load are known [1-3] and it is possible to find a reasonable calculation method [1] p. 344-360. Therefore, this paper only briefly discusses out-of-plane loaded inserts. The focus there is more on the proof of the failure mode with the digital image correlation technique.

In contrast, for in-plane loaded inserts it is not so easy to calculate the failure loads. The Insert Design Handbook (IDH) [1] provides a calculation rule for the maximum load in this direction. But this formula does not yield usable results for sandwich panels made of CFRP surface layers:

"The analysis method for in- plane load is based on test data. However, the results of these analyses do not correlate very well with the tests" [1] p. 192

Therefore, the focus of this paper is on the experimental determination of failure modes in in-plane loaded inserts. Due to the great variety of different sandwich materials and inserts, a small selection is investigated in this paper. Only 'through-the-thickness' inserts, blind inserts and special inserts potted in a sandwich with CFRP face sheets and a perforated aluminum honeycomb core are tested (see Figure 1).

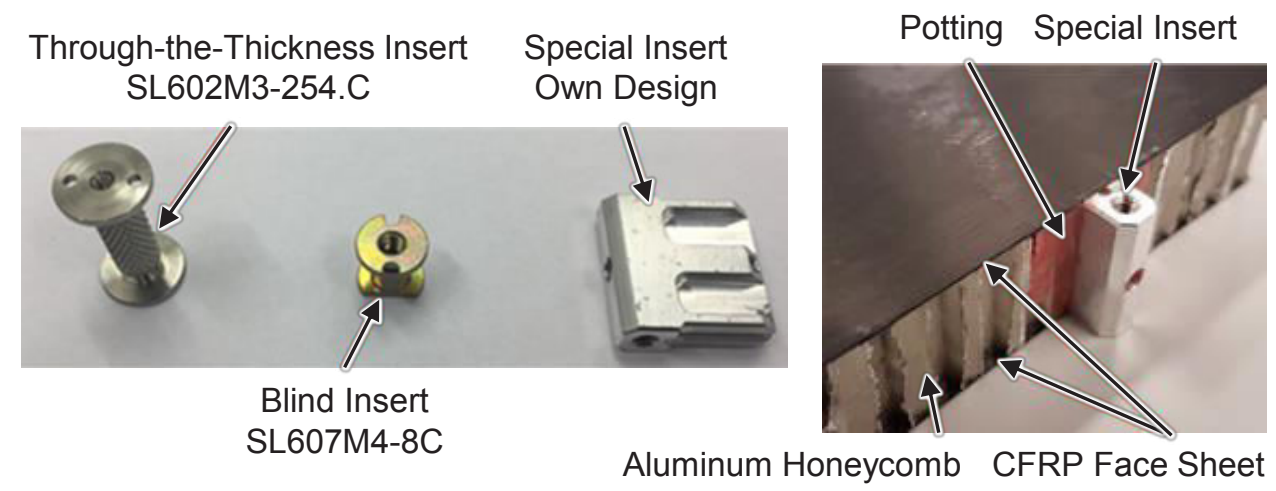

Figure 1: Investigated insert types and sample of a special insert potted in a CFRP sandwich 


\section{Specimen manufactures}

For the tests, 12 test specimens per standard insert type and 8 per special insert are produced. The sandwich's face sheets consist of four layers Torayca M55J / EX1515 UD laminate in the following orientation $[0, \pm 45,90$, Honeycomb, 90, $\pm 45,0]$. The PAMGXR1 perforated honeycomb made of Al 5052 has a cell size of $4.8 \mathrm{~mm}$ (3/16 inch). For potting the two components epoxy adhesive 3M EC 9323 is used. The standardized inserts are potted in $100 \mathrm{~mm} \times 100 \mathrm{~mm}$ sandwich panels in the middle. An adjustment tab ensures that the insert is plane to the surface. The adhesive is then injected through one of the holes until it comes out of the other hole. The special inserts are inserted and bonded in the front side of $100 \mathrm{~mm} \times 70 \mathrm{~mm}$ sandwich panels. To prevent the adhesive from running out during the curing process, the honeycomb core is taped around the insert. The adhesive is then pressed through the injection hole of the insert until it comes out through the reference groove at the top.

Before and after potting, the individual components are weighed. This is used to control the amount of potting material used in each specimen.

\section{Inserts under Out-of-Plane Load}

Even though the focus of this article is on in-plane loaded inserts, the failure behavior of out-of-plane inserts is briefly discussed. As mentioned in the introduction, the honeycomb core fails before the insert is pulled out of the sandwich [1-3]. However, this cannot be determined in retrospect, because after failure of the honeycomb core, the insert is still pulled out of the sandwich panel and thus also a (subsequent) failure of the top layer can be seen. To determine the actual reason for failure, the test must be stopped directly after the first drop in force and not, as is often the case with these tensile tests, after the force has dropped by a certain high percentage. Afterwards the sample is cut open and examined. The following out-of-plane tests are intended to check whether this failure can be proven with the help of digital image correlation.

In the case of the special inserts, the failure of the core could be proven well, as the honeycomb core at the insert can be looked at directly. This was shown in [4]. But it is much more difficult with standard inserts, as it is not possible to look directly into the honeycomb core around the insert.

The idea here is that the bending of the sandwich surface shortly before and after failure is compared at approximately the same force. If the core fails, the bending stiffness in this area decreases drastically, which must lead to a change in the bending line. This idea was investigated in a previous numerical calculation. The bending lines of the surface are determined in two numerical models. In one of these models, to create failure in the core near the insert, the mechanical properties of the core are set to virtually zero locally around the insert. Figure 5 shows that a significant change in the bending line can be observed.

Figure 2 shows the test setup for the out-of-plane tensile test. The insert is pulled over a screw, the sandwich is pressed against the test setup. The hole in the test setup has a diameter of $70 \mathrm{~mm}$ as recommended in the insert design handbook. During the test, the insert is pulled out at a speed of $1 \mathrm{~mm} / \mathrm{min}$. The Aramis system manufactured by gom $\mathrm{GmbH}$ is used to record the deformation of the surface. This DIC system has two cameras with which three-dimensional deformations can be recorded. Figure 3 shows the force deformation diagram of the through-the-thickness inserts, six samples were tested. 


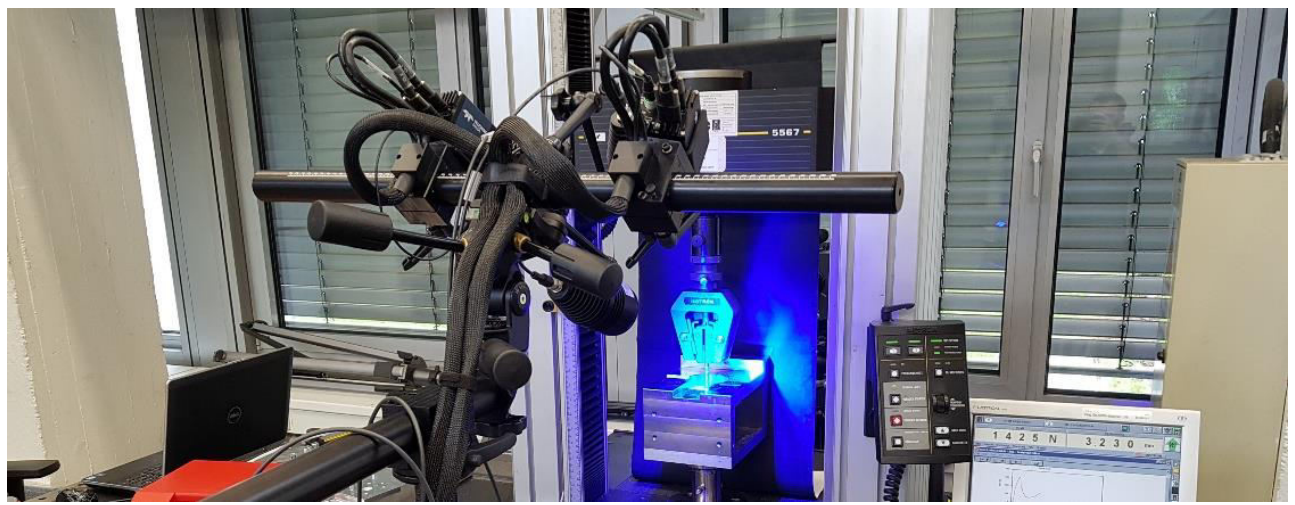

Figure 2: Out-of-plane test setup while using the Aramis System for Test monitoring

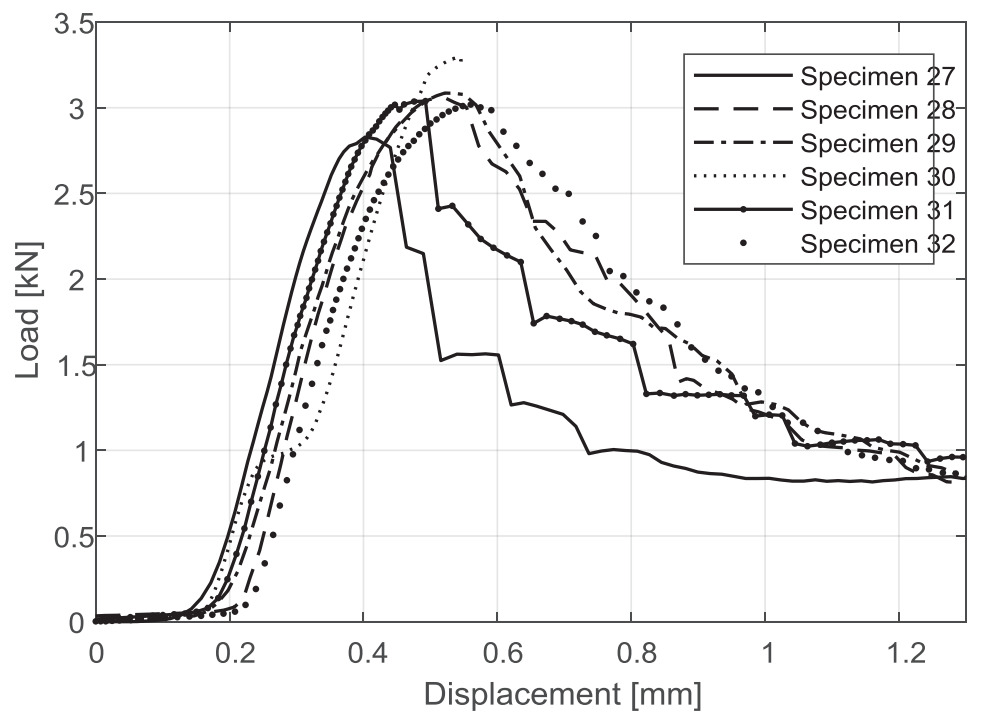

Figure 3: Load-displacement diagram of the through-the-thickness inserts out-of-plane test 
The investigation of the bending line before and after failure was carried out for all samples. Exemplary the deformation of sample 28 is discussed below. As can be seen in figure 4, the surface of the sandwich panel cannot be completely monitored with the Aramis system, because the test setup is in the way. Nevertheless, it is possible to adjust the system in such a way that a sufficient area could be taken up around a part of the bending line. The deformation of the surface is measured on the black line. Already when comparing the images monitored by Aramis before (figure 4 (a)) and after (figure 4 (b)) the failure it is obvious that the top layer around the insert is bent significantly more after failure. In addition, no failure can be detected in the surface course. This confirms that only the core fails first and not the top layer.

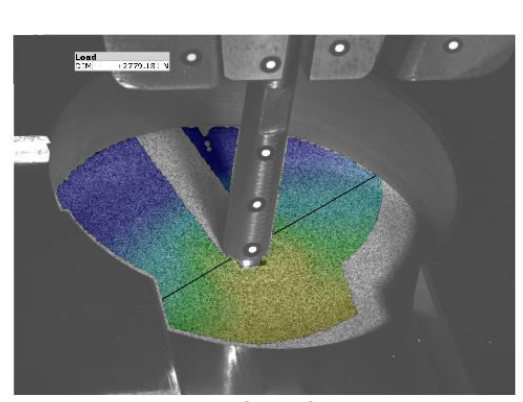

(a) Before failure

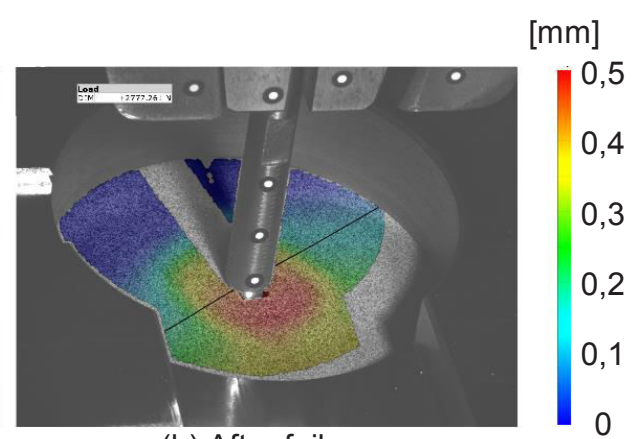

(b) After failure

Figure 4: Out-of-plane displacement of the sandwich surface before and after failure at the same load of specimen 28 
Figure 5 shows the different bending lines before and after the failure of the preliminary numerical study and the tested specimen 28 . It shows clearly that the bending stiffness decreases significantly due to the failure of the honeycomb core. When looking at the bending line, the area in which the honeycomb has failed can also be determined. The numerical model is generally a bit stiffer than the actual model. Otherwise the bending lines match very well. It can also be seen that plastic deformation has already taken place in the honeycomb core before failure. In the bending line from the failure determined by the test, a slight decrease in bending stiffness around the insert can be seen (compared to the numerically determined bending line). This is confirmed by the force deformation diagram. Sample 28 no longer deforms linearly at $2.8 \mathrm{kN}$. This non-linear range is also present in the other samples.

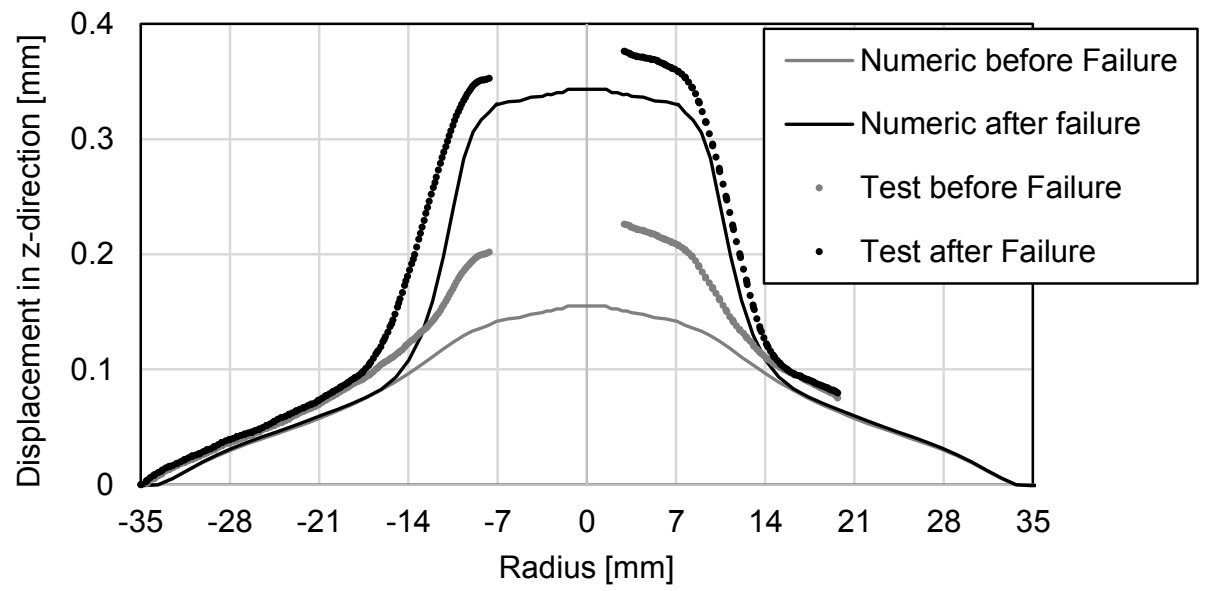

Figure 5: Comparison of the bending line of the sandwich surface before and after failure at a load of approximately $2.8 \mathrm{kN}$ of specimen 28

\section{Inserts under in-plane-load}

As already mentioned in the introduction, there are some difficulties with the strength calculation of inserts in CFRP sandwich panels loaded in the in-plane direction. This not only makes it difficult to design such connections in advance, but also requires many tests to be carried out. Looking at the formula provided in [1] p. 192, the strength calculation is like the calculation of bolts in a solid material. This makes sense as the insert is pressed against the surface layer during an in-plane test. It is therefore even more surprising that the formula provided does not reflect the calculation well. Tests are therefore carried out to find out whether failure in the top layer is really the cause of failure in standard inserts.

In addition, exit tests are carried out in in-plane Load by the newly developed inserts. As shown in [4], by using M3 screws, the screw fails and not the insert. To create a failure in the insert sandwich connection to determine the maximum carrying capacity, the inserts are drilled out so that the insert can be pulled out using a bolt.

\subsection{Standard inserts}




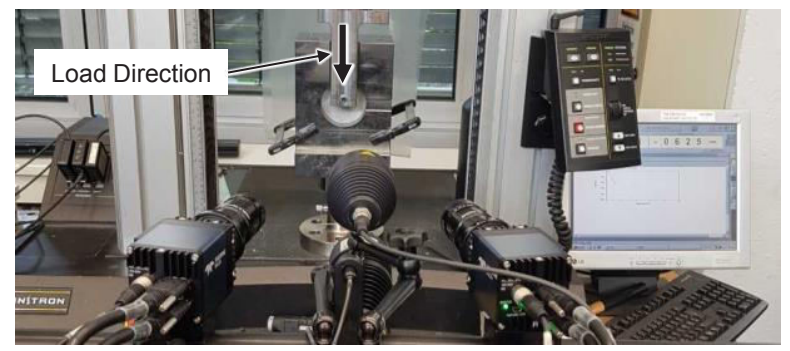

Figure 6: In-plane test setup while using the Aramis System for Test monitoring

For the in-plane tensile test, a test structure based on the structure presented in [1] was produced. The advantage of this is that the axis of force transmission lies exactly on the surface of the sandwich. This test is again monitored with Aramis, in which the surface is examined. Should a failure occur in the surface course, this should be taken up with the system. This test setup is shown in figure 6. Six specimen per insert type are tested.

The in-plane test results of the blind inserts and the trough-the-thickness inserts are shown in the figure 7 and 8 . With two samples in the through-the-thickness inserts, there were problems with the clamping (specimen 21 and 25), which is why the other four specimens (22-24 and 26) were drilled on to M4 to realize a connection with an M4 screw.

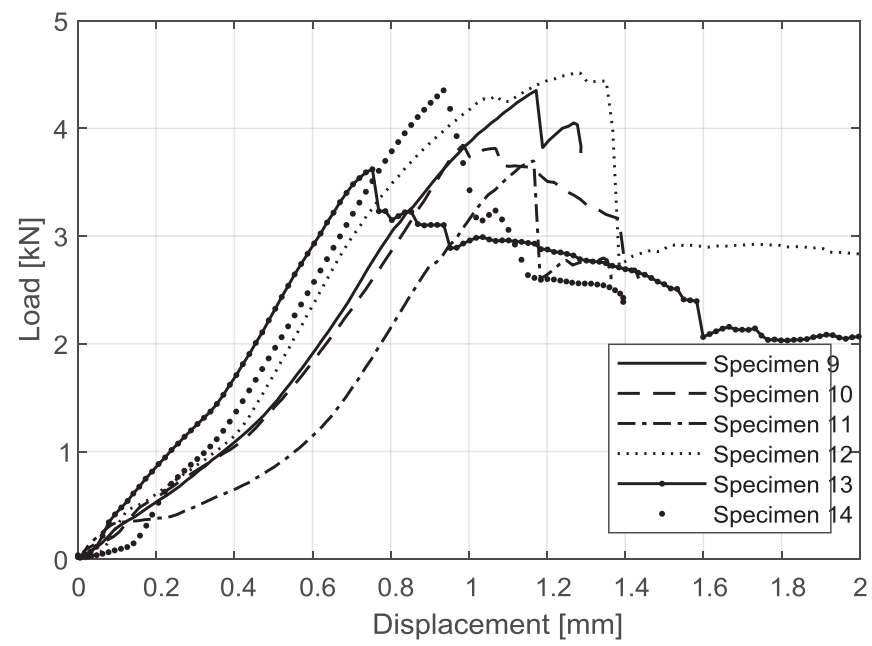

Figure 7: Load-displacement diagram of the blind inserts in-plane load test 


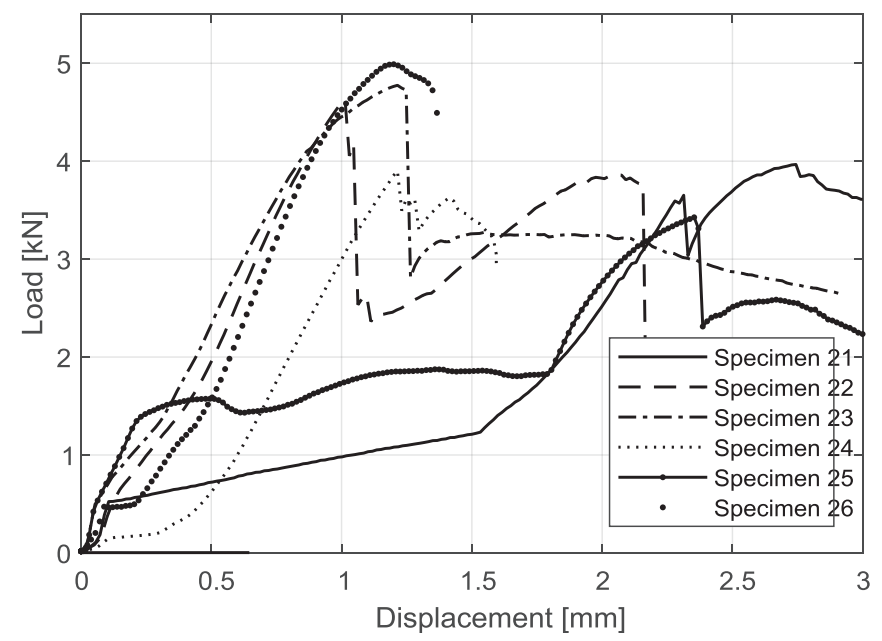

Figure 8: Load-displacement diagram of the through-the-thickness inserts in-plane load test

During the tests it was found that the surface course did not fail first. Some samples were immediately discontinued as soon as the first loss of strength was detected. No failure in the surface course could be detected during the examination of the latter. Therefore, the samples were cut open to examine the adhesive and honeycomb core. Figure 9 shows three of these insert connections. Two have a crack in the adhesive, one has an adhesive coating on the joint. The failure is always on the tension loaded side and not on the side against which the insert is pressed. This is probably caused by the force axis lying on the sandwich surface. As the insert with the adhesive protrudes deep into the sandwich panel, moments arise, resulting in the failure observed.

\section{Load Direction}
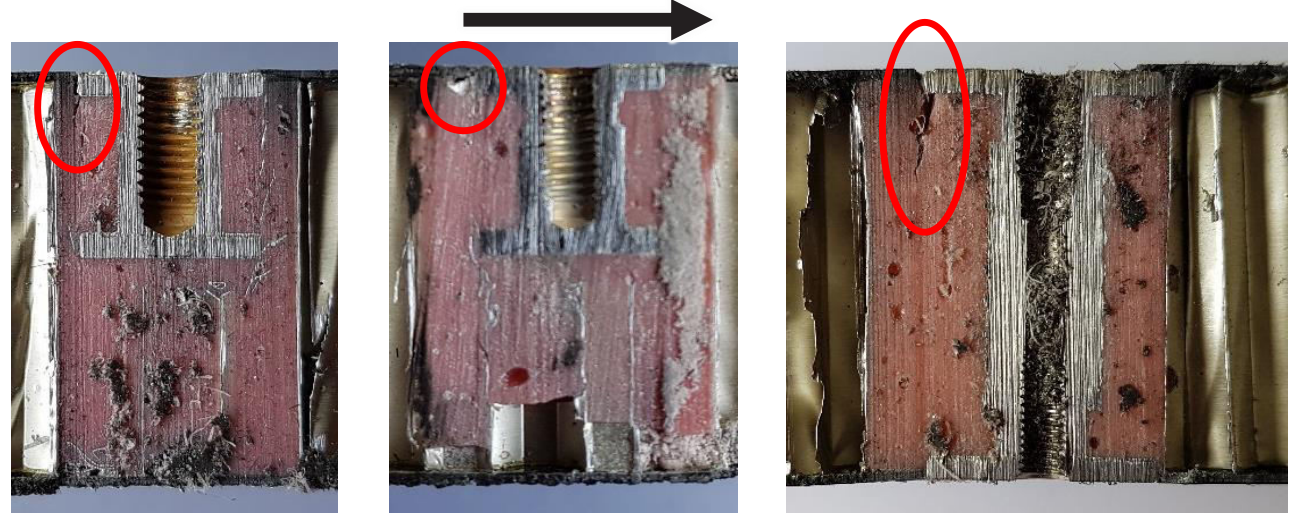

Figure 9: Initial failure in in-plane-loaded insert

\subsection{Special inserts}

For the in-plane tests of the special insert, the same test setup as presented in [5] is used. However, by using a bolt instead of screws, the inserts could be destroyed during the load 
test this time and thus the failure mode could be identified. The load displacement diagram of these tests and a tested specimen are shown in figure 10.
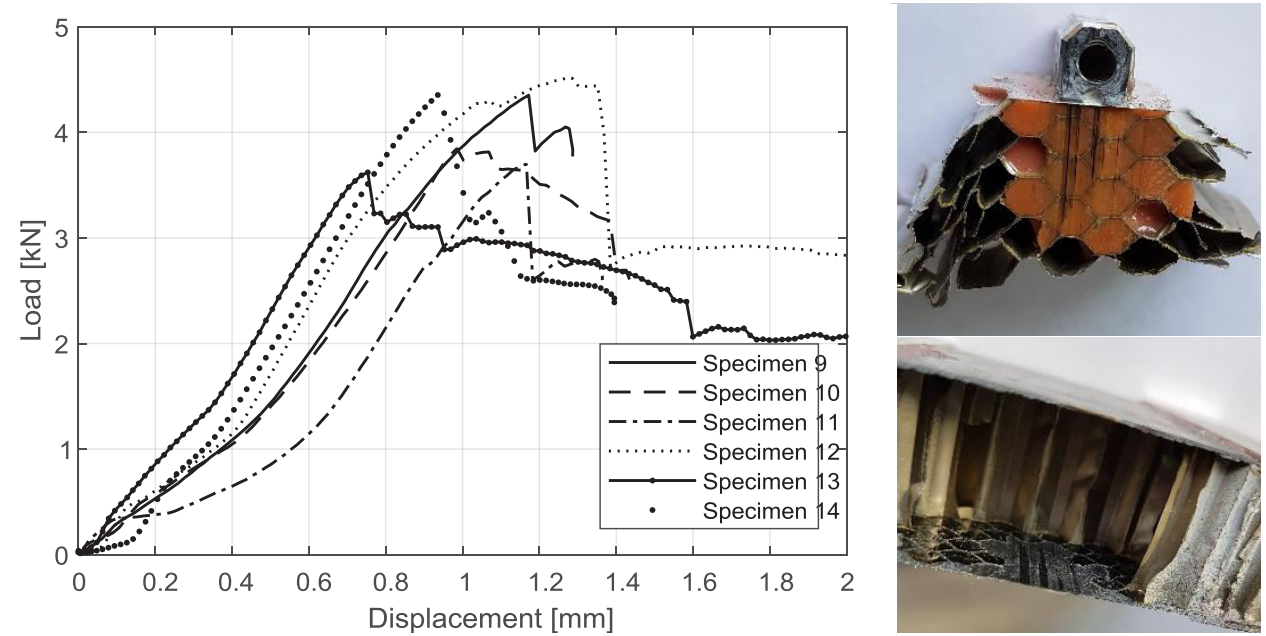

Figure 10: Load-displacement diagram of the special inserts in-plane load test and tested specimen

The tested specimens show all the same failure. The insert and the potting material is completely pulled out of the sandwich core. The connection between potting material and CFRP face sheet has failed, the connection of potting and insert is not damaged. To increase the load capacity in in-plane direction the inner side of the sandwich surfaces needs to be prepared.

\section{Summary and outlook}

This article showed that DIC can be used to measure core failure in out-of-plane tensile testing without subsequently destroying the specimen. It has also been demonstrated that the honeycomb is plastically deformed before failure. This is also visible in the force displacement diagram. In the in-plane test, it was shown that the adhesive in the tested joints first fails before the top layer is compressed. Here it is necessary to test further samples to investigate whether this behavior also occurs with other compounds. If this failure mode can also be proven for other insert connections, it may be possible to determine a new calculation approach for the load carrying capacity of in-plane loaded inserts. It is also interesting to test through-the-thickness insert on another test setup, where the insert is fixed from both sides. This causes the direction of tension to run through the middle of the sandwich, so that no moments occur in the insert potting compound.

The research leading to these results has received funding from the DLR Space Administration under grant agreement no. 50RA1502 by German Bundestag.

\section{References}

[1] ESA Requirements and Standards Division: Insert Design Handbook. ECSS-E-HB-3222,2011

[2] Song KI, Choi JY, Kweon JH, Choi JH and Kim KS: An experimental study of the insert joint strength of composite sandwich structures. Compos Structures 86: 107$113,2008$. 
[3] Heimbs, Sebastian and Pein, Marc: Failure behaviour of honeycomb sandwich corner joints and inserts. Composite Structures, 89: 575-588, 2009.

[4] P. Richert, J. Zimmermann, T. A. Schervan, A. Dafnis, K.-U. Schröder: Mechanical testing and analysis of hybrid bonded joints and load introduction elements. $15^{\text {th }}$ European Conference on Spacecraft Structures, Materials and Environmental Testing, Noordwijk, The Netherlands 